\title{
ON A CLASS OF NONLINEAR REACTION-DIFFUSION SYSTEMS WITH NONLOCAL BOUNDARY CONDITIONS
}

\author{
ABDELFATAH BOUZIANI
}

Received 30 July 2003

We prove the existence, uniqueness, and continuous dependence of a generalized solution of a nonlinear reaction-diffusion system with only integral terms in the boundaries. We first solve a particular case of the problem by using the energy-integral method. Next, via an iteration procedure, we derive the obtained results to study the solvability of the stated problem.

\section{Introduction}

In the recent years, a new attention has been given to reaction-diffusion systems which involve an integral over the spatial domain of a function of the desired solution on the boundary conditions; see $[2,5,7,6,9,10,17,18,19,20,22]$ and the references cited therein. Most of the studied problems in the current literature are devoted to problems which combine a classical boundary condition (Dirichlet, Neumann, etc.) with an integral condition for single linear equations. The purpose of this paper is to prove the existence and uniqueness of a solution for the following nonlinear reaction-diffusion system with only integral conditions:

$$
\begin{gathered}
\mathscr{L}_{1}(u, v)=\frac{\partial u}{\partial t}-\frac{\partial}{\partial x}\left(a_{1}(x, t) \frac{\partial u}{\partial x}\right)+b_{1}(x, t) v=f_{1}(x, t, u, v), \\
\mathscr{L}_{2}(u, v)=\frac{\partial v}{\partial t}-\frac{\partial}{\partial x}\left(a_{2}(x, t) \frac{\partial v}{\partial x}\right)+b_{2}(x, t) u=f_{2}(x, t, u, v), \\
\ell_{1} u=u(x, 0)=u_{0}(x), \\
\ell_{2} v=v(x, 0)=v_{0}(x), \\
\int_{\Omega} x^{k} u(x, t) d x=m_{k}(t) \quad(k=0,1), \\
\int_{\Omega} x^{k} v(x, t) d x=\mu_{k}(t) \quad(k=0,1),
\end{gathered}
$$

for $(x, t) \in Q$, where $Q=\Omega \times I, \Omega=(\alpha, \beta)$, is an open bounded interval of $\mathbb{R}, I=(0, T)$ with $0<T<+\infty$, and $f_{1}, f_{2}, u_{0}, v_{0}, m_{k}$, and $\mu_{k}(k=0,1)$ are known functions. 
Analogous systems with Dirichlet conditions have been studied in $[1,12,13,14,16]$. The existence of solutions have been obtained under the following assumptions:

(A1) the positivity of the solution is preserved with time, which is ensured by

$$
f_{1}(x, t, 0, v) \geq 0, \quad f_{1}(x, t, u, 0) \geq 0,
$$

for a.e. $(x, t) \in Q$, for all $u, v \geq 0$, and $u_{0}, v_{0} \geq 0$;

(A2) the total mass of the components $u, v$ is controlled with time, which is ensured by

$$
f_{1}+f_{2} \leq L_{1}(u+v+1) \quad \forall u, v \geq 0 \text {, a.e. }(x, t) \in Q
$$

(A3) the function $f_{1}$ verifies

$$
f_{1} \leq L_{2}(u+v+1) \quad \forall u, v \geq 0 \text {, a.e. }(x, t) \in Q \text {, }
$$

where $L_{1}$ and $L_{2}$ are positive constants.

However, in this paper, we will show the solvability of problem (1.1) without assuming conditions (A1), (A1), and (A3), and we will consider only the Lipschitz condition which will be explicitly given later.

The plan of the paper is as follows. In Section 2, we give some notations used throughout the paper. Section 3 is devoted to the solvability of a particular case of problem (1.1). In Section 3.1, we start by giving the statement of the problem. The concept of the solution we are considering is given in Section 3.2. Then we prove the uniqueness and continuous dependence in Section 3.3. Section 3.4 is reserved to the proof of the existence of the solution. In Section 4, we study problem (1.1). In Section 4.1, we reduce problem (1.1) to an equivalent form which is easier to analyze. The weak formulation of the reduced problem is given in Section 4.2. The existence of the solution is presented in Section 4.3. The uniqueness is established in Section 4.4, while the continuous dependence upon the data is treated in Section 4.5. Finally, we give a conclusion and more results on its generalization.

\section{Notation}

Let $L^{2}(\Omega)$ be the usual space of square integrable functions; its scalar product is denoted by $(\cdot, \cdot)$ and its associated norm by $\|\cdot\|$. We denote by $C_{0}(\Omega)$ the space of continuous functions with compact support in $\Omega$.

Definition 2.1. Denote by $B_{2}^{m}(\Omega)$ the Hilbert space defined as a completion of $C_{0}(\Omega)$ for the scalar product

$$
(z, w)_{B_{2}^{m}(\Omega)}:=\int_{\Omega} \mathfrak{J}_{x}^{m} z \cdot \mathfrak{J}_{x}^{m} w d x
$$


where $\mathfrak{J}_{x}^{m} z:=\int_{\alpha}^{x}\left((x-\xi)^{m-1} /(m-1) !\right) z(\xi) d \xi$. By the norm of function $z$ from $B_{2}^{m}(\Omega)$, the nonnegative number

$$
\|z\|_{B_{2}^{m}(\Omega)}:=\left(\int_{\Omega}\left(\mathfrak{J}_{x}^{m} z\right)^{2} d x\right)^{1 / 2}<\infty
$$

is understood.

Then, the inequality

$$
\|z\|_{B_{2}^{m}(\Omega)}^{2} \leq \frac{(\beta-\alpha)^{2}}{2}\|z\|_{B_{2}^{m-1}(\Omega)}^{2}, \quad m \geq 1,
$$

holds for every $z \in B_{2}^{m-1}(\Omega)$, and the embedding

$$
B_{2}^{m-1}(\Omega) \hookrightarrow B_{2}^{m}(\Omega)
$$

is continuous. If $m=0$, the space $B_{2}^{0}(\Omega)$ coincides with $L^{2}(\Omega)$.

Remark 2.2. Note that the space $B_{2}^{m}(\Omega)$ was first introduced by the author (see, e.g., $[2,3,7,8])$. It is a very useful space for this class of problems.

Definition 2.3. Denote by $L_{0}^{2}(\Omega)$ the space consisting of elements $z(x)$ of the space $L^{2}(\Omega)$ verifying $\int_{\Omega} x^{k} z(x) d x=0(k=0,1)$.

Remark 2.4. Since $L_{0}^{2}(\Omega)$ is the null space of the continuous linear mapping $\ell: L^{2}(\Omega) \rightarrow$ $\mathbb{R}^{2}, z \mapsto \ell(z)=\left(\int_{\Omega} z(x) d x, \int_{\Omega} x z(x) d x\right)$, it is a closed linear subspace of $L^{2}(\Omega)$; consequently, $L_{0}^{2}(\Omega)$ is a Hilbert space for $(\cdot, \cdot)$.

Let $X$ be a Hilbert space with a norm denoted by $\|\cdot\|_{X}$.

Definition 2.5. (i) Denote by $L^{2}(I ; X)$ the set of all measurable abstract functions $u(\cdot, t)$ from $I$ into $X$ such that

$$
\|u\|_{L^{2}(I ; X)}=\left(\int_{I}\|u(\cdot, t)\|_{X}^{2} d t\right)^{1 / 2}<\infty .
$$

(ii) Let $C(\bar{I} ; X)$ be the set of all continuous functions $u(\cdot, t): \bar{I} \rightarrow X$ with

$$
\|u\|_{C(\bar{I} ; X)}=\max _{t \in \bar{I}}\|u(\cdot, t)\|_{X}<\infty .
$$

We assume that

(A4) $0<c_{0} \leq a_{i} \leq c_{1},\left|\partial a_{i} / \partial t\right| \leq c_{2},\left|\partial a_{i} / \partial x\right| \leq c_{3},\left|b_{i}\right| \leq c_{4}(i=1,2)$ for all $(x, t) \in \bar{Q}$;

(A5) the functions $f_{i}(i=1,2)$ are bounded in $B_{2}^{1}(\Omega)$ and fulfill the Lipschitz condition, that is, there exists a positive constant $L$ such that

$$
\left\|f_{i}\left(\cdot, t, p_{1}, q_{1}\right)-f_{i}\left(\cdot, t, p_{2}, q_{2}\right)\right\|_{B_{2}^{1}(\Omega)} \leq L\left(\left\|p_{1}-p_{2}\right\|_{B_{2}^{1}(\Omega)}+\left\|q_{1}-q_{2}\right\|_{B_{2}^{1}(\Omega)}\right) ;
$$

(A6) we have the following compatibility conditions:

$$
\int_{\Omega} x^{k} u_{0}(x) d x=m_{k}(0), \quad \int_{\Omega} x^{k} v_{0}(x) d x=\mu_{k}(0) \quad(k=0,1) .
$$




\section{A particular case}

3.1. Statement of the problem. In this section, we deal with a particular case of problem (1.1) in which the system is reduced to a single linear equation related to the first component. Precisely, we consider the problem of finding a function $u=u(x, t)$ satisfying

$$
\begin{gathered}
\mathscr{L} u=\frac{\partial u}{\partial t}-\frac{\partial}{\partial x}\left(a(x, t) \frac{\partial u}{\partial x}\right)+b(x, t) u=f(x, t), \quad(x, t) \in Q \\
\ell u=u(x, 0)=u_{0}(x), \quad x \in \Omega, \\
\int_{\Omega} x^{k} u(x, t) d x=m_{k}(t) \quad(k=0,1), t \in I
\end{gathered}
$$

with

$$
\int_{\Omega} x^{k} u_{0}(x) d x=m_{k}(0) \quad(k=0,1)
$$

where functions $a$ and $b$ verify the same assumptions on $a_{i}$ and $b_{i}$, respectively, given in (A4). Problem (3.1) arises from some practical phenomena such as the identification of the entropy in the quasistatic flexure of a thermoelastic rod [7].

We start by reducing problem (3.1) with inhomogeneous integral conditions to an equivalent problem with homogeneous conditions. In order to achieve this, we introduce a new unknown function $z$ defined by $z(x, t)=u(x, t)-U(x, t)$, where

$$
\begin{aligned}
U(x, t)= & \frac{\left((\beta-\alpha)^{3}+12\left(\beta^{2}-\alpha^{2}\right)(x-\alpha)-18(\beta+\alpha)(x-\alpha)^{2}\right)}{(\beta-\alpha)^{4}} m_{0}(t) \\
& +\frac{12\left(3(x-\alpha)^{2}-2(\beta-\alpha)(x-\alpha)\right)}{(\beta-\alpha)^{4}} m_{1}(t) .
\end{aligned}
$$

Therefore, problem (3.1) becomes

$$
\begin{gathered}
\mathscr{L} z=\frac{\partial z}{\partial t}-\frac{\partial}{\partial x}\left(a(x, t) \frac{\partial z}{\partial x}\right)+b(x, t) z=f(x, t), \quad(x, t) \in Q \\
\ell z=z(x, 0)=z_{0}(x), \quad x \in \Omega \\
\int_{\Omega} x^{k} z(x, t) d x=0, \quad k=0,1, t \in I
\end{gathered}
$$

with

$$
\int_{\Omega} x^{k} z_{0}(x) d x=0, \quad k=0,1
$$

where $f(x, t)=\mathrm{f}(x, t)-\mathscr{L} U$ and $z_{0}(x)=u_{0}(x)-U(x, 0)$. 
3.2. A strongly generalized formulation. In this section, we make precise the concept of the solution of problem (3.4) we are considering.

Following the energy-integral method [21], we reformulate problem (3.4) as the problem of solving the operator equation

$$
L z=\left\{f, z_{0}\right\}
$$

where $L$ is an unbounded operator which maps $z(x, t)$ to the pair of elements $\mathscr{L} z$ and $\ell z$ so that $L=\{\mathscr{L}, \ell\}$. The operator $L$, with domain $D(L)$, acts from the space $B$ into the space $F$, where $D(L)$ is the set of all elements $z$ for which $\partial z / \partial t, \partial z / \partial x, \partial^{2} z / \partial x^{2} \in L^{2}\left(I ; L^{2}(\Omega)\right)$ and satisfying conditions (3.4c), $B$ is a Banach space obtained by enclosing the set $D(L)$ with respect to the norm

$$
\|z\|_{B}=\left(\left\|\frac{\partial z}{\partial t}\right\|_{L^{2}\left(I ; B_{2}^{1}(\Omega)\right)}^{2}+\|z\|_{C\left(\bar{I} ; L^{2}(\Omega)\right)}^{2}\right)^{1 / 2},
$$

and $F$ is the Hilbert space $L^{2}\left(I ; L^{2}(\Omega)\right) \times L^{2}(\Omega)$ consisting of all elements $\left\{f, z_{0}\right\}$ for which the norm

$$
\left\|\left\{f, z_{0}\right\}\right\|_{F}^{2}=\|f\|_{L^{2}\left(I ; L^{2}(\Omega)\right)}^{2}+\left\|z_{0}\right\|^{2}
$$

is finite. We denote by $\bar{L}$ the closure of $L$, and by $D(\bar{L})$, the domain of $\bar{L}$.

Definition 3.1. The solution of the operator equation

$$
\bar{L} z=\left\{f, z_{0}\right\}
$$

is called a strongly generalized solution of problem (3.4) or of equation (3.6).

Remark 3.2. A strongly generalized solution in the sense of Definition 3.1 is also a weak solution (see, e.g., [11]).

3.3. Uniqueness and continuous dependence on the solution. In this section, we establish an energy inequality for $L$. Then, uniqueness and continuous dependence are a direct corollary of it.

TheOREm 3.3. Under assumption (A1), there exists some positive constant $C$ independent of $z$ such that

$$
\|z\|_{B} \leq C\|L z\|_{F} .
$$

Proof. Considering the scalar product in $B_{2}^{1}(\Omega)$ of $(3.4 \mathrm{a})$ and $\partial z / \partial t$, and integrating the result over $(0, \tau)$ with $0 \leq \tau \leq T$, we have

$$
\begin{aligned}
\int_{0}^{\tau} \| & \frac{\partial z(\cdot, t)}{\partial t} \|_{B_{2}^{1}(\Omega)}^{2} d t-\int_{0}^{\tau}\left(\frac{\partial}{\partial x}\left(a \frac{\partial z(\cdot, t)}{\partial x}\right), \frac{\partial z(\cdot, t)}{\partial t}\right)_{B_{2}^{1}(\Omega)} d t \\
& +\int_{0}^{\tau}\left(b z(\cdot, t), \frac{\partial z(\cdot, t)}{\partial t}\right)_{B_{2}^{1}(\Omega)} d t=\int_{0}^{\tau}\left(f(\cdot, t), \frac{\partial z(\cdot, t)}{\partial t}\right)_{B_{2}^{1}(\Omega)} d t .
\end{aligned}
$$


From integration by parts, we know that

$$
\begin{aligned}
-\int_{0}^{\tau}( & \left.\frac{\partial}{\partial x}\left(a \frac{\partial z(\cdot, t)}{\partial x}\right), \frac{\partial z(\cdot, t)}{\partial t}\right)_{B_{2}^{1}(\Omega)} d t \\
= & \frac{1}{2} \int_{\Omega} a(x, \tau)(z(x, \tau))^{2} d x-\frac{1}{2} \int_{\Omega} a(x, 0)\left(z_{0}(x)\right)^{2} d x \\
& -\frac{1}{2} \int_{0}^{\tau} \int_{\Omega} \frac{\partial a(x, t)}{\partial t} z^{2} d x d t+\int_{0}^{\tau}\left(\frac{\partial a}{\partial x} z(\cdot, t), \mathfrak{J}_{x} \frac{\partial z(\cdot, t)}{\partial t}\right) d t .
\end{aligned}
$$

So, it is easy to see that

$$
\begin{aligned}
2 \int_{0}^{\tau} & \left\|\frac{\partial z(\cdot, t)}{\partial t}\right\|_{B_{2}^{1}(\Omega)}^{2} d t+\int_{\Omega} a(x, \tau)(z(x, \tau))^{2} d x \\
= & 2 \int_{0}^{\tau}\left(f(\cdot, t), \frac{\partial z(\cdot, t)}{\partial t}\right)_{B_{2}^{1}(\Omega)} d t+\int_{\Omega} a(x, 0)\left(z_{0}(x)\right)^{2} d x \\
& +\int_{0}^{\tau} \int_{\Omega}^{\tau} \frac{\partial a(x, t)}{\partial t} z^{2} d x d t-2 \int_{0}^{\tau}\left(\frac{\partial a}{\partial x} z(\cdot, t), \mathfrak{J}_{x} \frac{\partial z(\cdot, t)}{\partial t}\right) d t \\
& -2 \int_{0}^{\tau}\left(b z(\cdot, t), \frac{\partial z(\cdot, t)}{\partial t}\right)_{B_{2}^{1}(\Omega)} d t .
\end{aligned}
$$

Taking into account (A4) and applying the Cauchy inequality to the first and the last two terms on the right-hand side of (3.13), we obtain

$$
\begin{aligned}
& \int_{0}^{\tau}\left\|\frac{\partial z(\cdot, t)}{\partial t}\right\|_{B_{2}^{1}(\Omega)}^{2} d t+\|z(\cdot, \tau)\|^{2} \\
& \quad \leq c_{5}\left(\int_{0}^{\tau}\|f(\cdot, t)\|^{2} d t+\left\|z_{0}\right\|^{2}\right)+c_{6} \int_{0}^{\tau}\|z(\cdot, t)\|^{2} d t
\end{aligned}
$$

where $c_{5}=\max \left((\beta-\alpha)^{2}, c_{1}\right) / \min \left(1 / 2, c_{0}\right)$ and $c_{6}=\max \left(c_{2}+2 c_{3}^{2}+(\beta-\alpha)^{2} c_{4}^{2}\right) / \min \left(1 / 2, c_{0}\right)$. Using a lemma of Gronwall's type to eliminate the last integral on the right-hand side of (3.14), it yields

$$
\int_{0}^{\tau}\left\|\frac{\partial z(\cdot, t)}{\partial t}\right\|_{B_{2}^{1}(\Omega)}^{2} d t+\|z(\cdot, \tau)\|^{2} \leq c_{5} \exp \left(c_{6} T\right)\left(\int_{0}^{T}\|f(\cdot, t)\|^{2} d t+\left\|z_{0}\right\|^{2}\right)
$$

Since the right-hand side of (3.15) is independent of $\tau$, we replace the left-hand side by the upper bound with respect to $\tau$ from 0 to $T$. Thus inequality (3.10) holds, with $C=$ $c_{5}^{1 / 2} \exp \left(c_{6} T / 2\right)$.

Proposition 3.4. The operator $L: B \rightarrow F$ possesses a closure.

For the proof, we refer the reader to [6]. 
Since the points of the graph of $\bar{L}$ are limits of sequences of points of the graph of $L$, inequality (3.10) can be extended for operator $\bar{L}$, that is,

$$
\|z\|_{B} \leq C\|\bar{L} z\|_{F} \quad \forall u \in D(\bar{L})
$$

from which we have the following results.

Corollary 3.5. The strongly generalized solution of problem (2.4) if it exists is unique and depends continuously on $\left(f, u_{0}\right) \in F$.

Proof. This can be obtained directly from estimate (3.16).

Corollary 3.6. The range $R(\bar{L})$ of $\bar{L}$ is closed in $F$ and $R(\bar{L})=\overline{R(L)}$.

Proof. Similar to that in [6].

3.4. Existence of the solution. We now prove the existence of the solution of problem (3.4) in the sense of Definition 3.1.

Theorem 3.7. Let assumption (A4) be satisfied. Then for any $f \in L^{2}\left(I ; L^{2}(\Omega)\right)$ and $z_{0} \in$ $L^{2}(\Omega)$, there exists a unique strongly generalized solution $z=\bar{L}^{-1}\left\{f, z_{0}\right\}=\bar{L}^{-1}\left\{f, z_{0}\right\}$ of problem (3.4).

Proof. It follows from Corollary 3.6 that to prove the existence of the solution, it remains to show that $R(L)$ is everywhere dense in $F$. To this end, we first establish the density for the special case when the operator $L$ is reduced to $L_{0}$ with domain $D\left(L_{0}\right)=D(L)$, where $L_{0} z=\left(\mathscr{L}_{0} z, \ell z\right), \mathscr{L}_{0}$ is the principal part of $\mathscr{L}$, that is,

$$
\mathscr{L}_{0} z=\frac{\partial z}{\partial t}-\frac{\partial}{\partial x}\left(a(x, t) \frac{\partial z}{\partial x}\right)
$$

Proposition 3.8. Let the assumptions of Theorem 3.7 hold. If

$$
\left(\mathscr{L}_{0} z, \omega\right)_{L^{2}\left(I ; L^{2}(\Omega)\right)}=0
$$

for some $\omega \in L^{2}\left(I ; L^{2}(\Omega)\right)$ and for all $u \in D_{0}\left(L_{0}\right)=\left\{z \in D\left(L_{0}\right): \ell z=0\right\}$, then $\omega$ vanishes almost everywhere in $\mathrm{Q}$.

We assume for the moment that the proof of Proposition 3.8 has been established, and return to the proof of Theorem 3.7.

Suppose that for some $W=\left(\omega, \omega_{0}\right) \in F$ and all $z \in D\left(L_{0}\right)$,

$$
\left(\mathscr{L}_{0} z, \omega\right)_{L^{2}\left(I ; L^{2}(\Omega)\right)}+\left(\ell z, \omega_{0}\right)=0 .
$$

We must prove that $W \equiv 0$. To this end, putting $z \in D_{0}\left(L_{0}\right)$ in the last equality, we obtain (3.18). Hence, Proposition 3.8 implies that $\omega \equiv 0$. Thus it follows that

$$
\left(\ell z, \omega_{0}\right)=0, \quad z \in D\left(L_{0}\right) .
$$

Since the range $R(\ell)$ is dense in $L^{2}(\Omega)$, the last equality implies that $\omega_{0} \equiv 0$. Hence, $W \equiv 0$. 
The general result may be derived by means of the continuity method with respect to the parameter (see, for instance, [5, Proof of Theorem 3]).

To complete the proof of Theorem 3.7, it remains to prove Proposition 3.8.

Proof of Proposition 3.8. Identity (3.18) can be written in the form

$$
\left(\frac{\partial z}{\partial t}, \omega\right)_{L^{2}\left(I ; L^{2}(\Omega)\right)}=\left(\frac{\partial}{\partial x}\left(a(x, t) \frac{\partial z}{\partial x}\right), \omega\right)_{L^{2}\left(I ; L^{2}(\Omega)\right)} .
$$

In equality (3.21), we put

$$
z=\int_{0}^{t} e^{c \tau} y(x, \tau) d \tau
$$

where $c$ is a constant such that

$$
c c_{0}-c_{2}-c_{3}^{2} \geq 0
$$

$\mathfrak{J}_{t}\left(e^{\mathcal{c} \tau} y\right), \partial \mathfrak{J}_{t}\left(e^{c \tau} y\right) / \partial x,(\partial / \partial x)\left(a(x, t)\left(\partial \mathfrak{J}_{t}\left(e^{c \tau} y\right) / \partial x\right)\right)$ are in $L^{2}\left(I ; L^{2}(\Omega)\right)$, and $y$ satisfy conditions (3.4c).

Substituting (3.22) into (3.21), we have

$$
\left(e^{c t} y, \omega\right)_{L^{2}\left(I ; L^{2}(\Omega)\right)}=\left(\frac{\partial}{\partial x}\left(a \frac{\partial \mathfrak{J}_{t}\left(e^{c \tau} y\right)}{\partial x}\right), \omega\right)_{L^{2}\left(I ; L^{2}(\Omega)\right)} .
$$

The left-hand side of equality (3.24) shows that the mapping

$$
L^{2}\left(I ; L^{2}(\Omega)\right) \ni z \longrightarrow \frac{\partial}{\partial x}\left(a(x, t) \frac{\partial \mathfrak{J}_{t}\left(e^{c \tau} y\right)}{\partial x}\right)
$$

is continuous if the function $\omega$ on the right-hand side of (3.24) verifies $a(\partial \omega / \partial x)$, $\mathfrak{J}_{t}^{*}(a(\partial \omega / \partial x)),(\partial / \partial x)\left(\mathfrak{J}_{t}^{*}(a(\partial \omega / \partial x))\right) \in L^{2}\left(I ; L^{2}(\Omega)\right)$ such that

$$
\left.\omega\right|_{x=\alpha}=\left.\omega\right|_{x=\beta}=\left.\frac{\partial \omega}{\partial x}\right|_{x=\alpha}=\left.\frac{\partial \omega}{\partial x}\right|_{x=\beta}=0
$$

Set

$$
\omega=-2 \mathfrak{J}_{x}^{2} y
$$

It is easy to see that $\omega$ defined in (3.27) verifies conditions (3.26). We now substitute (3.27) into (3.24), and we get

$$
-2\left(e^{c t} y, \mathfrak{J}_{x}^{2} y\right)_{L^{2}\left(I ; L^{2}(\Omega)\right)}=-2\left(\frac{\partial}{\partial x}\left(a \frac{\partial \mathfrak{J}_{t}\left(e^{\mathcal{c} \tau} y\right)}{\partial x}\right), \mathfrak{J}_{x}^{2} y\right)_{L^{2}\left(I ; L^{2}(\Omega)\right)}
$$


Integrating by parts each term of (3.28) by taking into account conditions (3.4c), we have

$$
\begin{aligned}
& -2\left(e^{c t} y, \mathfrak{I}_{x}^{2} y\right)_{L^{2}\left(I ; L^{2}(\Omega)\right)}=2 \int_{Q} e^{c t}\left(\mathfrak{I}_{x} y\right)^{2} d x d t \\
& -2\left(\frac{\partial}{\partial x}\left(a \frac{\partial \mathfrak{I}_{t}\left(e^{c \tau} y\right)}{\partial x}\right), \mathfrak{J}_{x}^{2} y\right)_{L^{2}\left(I ; L^{2}(\Omega)\right)}=-\int_{\Omega} e^{-c T} a(x, T)\left(\mathfrak{I}_{T}\left(e^{c t} y\right)\right)^{2} d x \\
& \quad-\int_{Q} e^{-c t}\left(c a-\frac{\partial a}{\partial t}\right)\left(\mathfrak{I}_{t}\left(e^{c \tau} y\right)\right)^{2} d x d t-2 \int_{Q} \frac{\partial a}{\partial x} \mathfrak{I}_{t}\left(e^{c \tau} y\right) \mathfrak{I}_{x} y d x d t \\
& \leq-\int_{\Omega} e^{-c T} a(x, T)\left(\mathfrak{I}_{T}\left(e^{c t} y\right)\right)^{2} d x+\int_{Q} e^{c t}\left(\mathfrak{I}_{x} y\right)^{2} d x d t \\
& -\int_{Q} e^{-c t}\left(c a-\frac{\partial a}{\partial t}-\left(\frac{\partial a}{\partial x}\right)^{2}\right)\left(\mathfrak{I}_{t}\left(e^{c \tau} y\right)\right)^{2} d x d t
\end{aligned}
$$

Substituting (3.29) and (3.30) into (3.28) yields

$$
\begin{aligned}
\int_{Q} e^{c t}\left(\mathfrak{J}_{x} y\right)^{2} d x d t \leq & -\int_{\Omega} e^{-c T} a(x, T)\left(\mathfrak{J}_{T}\left(e^{c t} y\right)\right)^{2} d x \\
& -\int_{Q} e^{-c t}\left(c a-\frac{\partial a}{\partial t}-\left(\frac{\partial a}{\partial x}\right)^{2}\right)\left(\mathfrak{J}_{t}\left(e^{c \tau} y\right)\right)^{2} d x d t .
\end{aligned}
$$

We now utilize assumption (A4) and discard the first integral on the right-hand side of (3.31); we get

$$
\int_{Q} e^{c t}\left(\mathfrak{J}_{x} y\right)^{2} d x d t \leq-\left(c c_{0}-c_{2}-c_{3}^{2}\right) \int_{Q} e^{-c t}\left(\mathfrak{I}_{t}\left(e^{c \tau} y\right)\right)^{2} d x d t
$$

By virtue of condition (3.23), we conclude that

$$
\int_{Q} e^{c t}\left(\mathfrak{J}_{x} y\right)^{2} d x d t \leq 0
$$

and thus $\mathfrak{J}_{x} y \equiv 0$. Hence, $\omega \equiv 0$, and this completes the proof of Theorem 3.7.

\section{The general case}

4.1. An equivalent problem. We consider a particular case of problem (1.1) in which we set $f_{2}=-f_{1}, a_{1}(x, t)=a_{2}(x, t), b_{1}(x, t)=b_{2}(x, t)$, and $w=u+v$. Thus, we obtain the following problem:

$$
\begin{gathered}
\frac{\partial w}{\partial t}-\frac{\partial}{\partial x}\left(a_{1}(x, t) \frac{\partial w}{\partial x}\right)+b_{1}(x, t) w=0, \quad(x, t) \in Q \\
w(x, 0)=w_{0}(x), \quad x \in \Omega, \\
\int_{\Omega} x^{k} w(x, t) d x=M_{k}(t), \quad(k=0,1), t \in I,
\end{gathered}
$$


where $M_{k}(t)=m_{k}(t)+\mu_{k}(t)$. From the previous section, we deduce that problem (4.1) possesses a unique solution that continuously depends on the data. Then, the function $u=u(x, t)$ of problem (1.1) satisfies

$$
\begin{gathered}
\mathscr{L}_{1} u=\frac{\partial u}{\partial t}-\frac{\partial}{\partial x}\left(a_{1} \frac{\partial u}{\partial x}\right)-b_{1} u=f_{3}(x, t, u), \quad(x, t) \in Q, \\
\ell_{1} u=u(x, 0)=u_{0}(x), \quad x \in \Omega, \\
\int_{\Omega} x^{k} u(x, t) d x=m_{k}(t), \quad(k=0,1), t \in I,
\end{gathered}
$$

where $f_{3}(x, t, u)=f_{1}(x, t, u, w-u)-b_{1}(x, t) w$. Therefore, solving (1.1) is equivalent to solving (4.2) and to set

$$
v=w-u .
$$

We introduce a new function $\sigma=\sigma(x, t)$ verifying $\sigma(x, t)=u(x, t)-U(x, t)$, where $U(x, t)$ is defined by (3.3). Consequently, the function $\sigma(x, t)$ will be defined as the solution of

$$
\begin{gathered}
\mathscr{L}_{1} \sigma=\frac{\partial \sigma}{\partial t}-\frac{\partial}{\partial x}\left(a_{1} \frac{\partial \sigma}{\partial x}\right)-b_{1} \sigma=f_{4}(x, t, \sigma), \quad(x, t) \in Q \\
\ell_{1} \sigma=\sigma(x, 0)=\sigma_{0}(x), \quad x \in \Omega, \\
\int_{\Omega} x^{k} \sigma(x, t) d x=0, \quad(k=0,1), t \in I,
\end{gathered}
$$

where $f_{4}(x, t, \sigma)=f_{3}(x, t, \sigma+U)+\mathscr{L}_{1} U, \sigma_{0}(x)=u_{0}(x)-U(x, 0)$. We introduce the auxiliary problem

$$
\begin{gathered}
\mathscr{L}_{1} \eta=\frac{\partial \eta}{\partial t}-\frac{\partial}{\partial x}\left(a_{1}(x, t) \frac{\partial \eta}{\partial x}\right)-b_{1}(x, t) \eta=0, \\
\ell_{1} \eta=\eta(x, 0)=\sigma_{0}(x), \\
\int_{\Omega} x^{k} \eta(x, t) d x=0 \quad(k=0,1)
\end{gathered}
$$

which we know from the previous section that it admits a unique solution depending upon the initial condition. Set $\theta(x, t)=\sigma(x, t)-\eta(x, t)$. Then $\theta(x, t)$ satisfies

$$
\begin{gathered}
\mathscr{L}_{1} \theta=\frac{\partial \theta}{\partial t}-\frac{\partial}{\partial x}\left(a \frac{\partial \theta}{\partial x}\right)-b_{1} \theta=f(x, t, \theta), \quad(x, t) \in Q, \\
\ell_{1} \theta=\theta(x, 0)=0, \quad x \in \Omega, \\
\int_{\Omega} x^{k} \theta(x, t) d x=0, \quad(k=0,1), t \in I,
\end{gathered}
$$

where $f(x, t, \theta)=f_{4}(x, t, \theta+\eta)$. Thus to prove the solvability of problem (1.1), it remains to establish the proof for problem (4.6).

As a function of kind $f_{i}(i=1,2)$, the function $f$ verifies the following assumption:

$\left(\mathrm{A} 5^{\prime}\right)$ the function $f$ is bounded in $B_{2}^{1}(\Omega)$ and satisfies the Lipschitz condition, that is, there exists a positive constant $L$ such that $\|f(\cdot, t, p)-f(\cdot, t, q)\|_{B_{2}^{1}(\Omega)} \leq L \| p-$ $q \|_{B_{2}^{1}(\Omega)}$. 
4.2. A weakly generalized formulation. Considering the scalar product in $B_{2}^{1}(\Omega)$ of (4.6a) and $\omega(\cdot, t) \in L_{0}^{2}(\Omega)$, it yields

$$
\begin{gathered}
\left(\frac{\partial \theta(\cdot, t)}{\partial t}, \omega(\cdot, t)\right)_{B_{2}^{1}(\Omega)}-\left(\frac{\partial}{\partial x}\left(a_{1} \frac{\partial \theta(\cdot, t)}{\partial x}\right), \omega(\cdot, t)\right)_{B_{2}^{1}(\Omega)}-\left(b_{1} \theta(\cdot, t), \omega(\cdot, t)\right)_{B_{2}^{1}(\Omega)} \\
=(f(\cdot, t, \theta(\cdot, t)), \omega(\cdot, t))_{B_{2}^{1}(\Omega)} \quad \forall t \in I .
\end{gathered}
$$

Integrating by parts the second term on the left-hand side of (4.7),

$$
\begin{aligned}
& -\left(\frac{\partial}{\partial x}\left(a_{1} \frac{\partial \theta(\cdot, t)}{\partial x}\right), \oplus(\cdot, t)\right)_{B_{2}^{1}(\Omega)} \\
& \quad=-\left(\mathfrak{J}_{x} \frac{\partial}{\partial \xi}\left(a_{1} \frac{\partial \theta(\cdot, t)}{\partial \xi}\right), \mathfrak{J}_{x} \oplus(\cdot, t)\right) \\
& \quad=-\left.\mathfrak{J}_{x} \frac{\partial}{\partial \xi}\left(a_{1} \frac{\partial \theta}{\partial \xi}\right) \cdot \mathfrak{J}_{x}^{2} \omega\right|_{\alpha} ^{\beta}+\left(\frac{\partial}{\partial x}\left(a_{1} \frac{\partial \theta(\cdot, t)}{\partial x}\right), \mathfrak{J}_{x}^{2} \omega(\cdot, t)\right) \\
& \quad=\left.a_{1} \frac{\partial \theta}{\partial x} \cdot \mathfrak{J}_{x}^{2} \omega\right|_{\alpha} ^{\beta}-\left(a_{1} \frac{\partial \theta(\cdot, t)}{\partial x}, \mathfrak{J}_{x} \oplus(\cdot, t)\right) \\
& \quad=-\left.a_{1} \theta \cdot \mathfrak{J}_{x} \omega\right|_{\alpha} ^{\beta}+\left(a_{1} \theta(\cdot, t), \oplus(\cdot, t)\right)+\left(\frac{\partial a_{1}}{\partial x} \theta(\cdot, t), \mathfrak{J}_{x} \omega(\cdot, t)\right),
\end{aligned}
$$

from which we obtain

$$
\begin{aligned}
\left(\frac{\partial \theta(\cdot, t)}{\partial t}, \varpi(\cdot, t)\right)_{B_{2}^{1}(\Omega)}+\left(a_{1} \theta(\cdot, t), \varpi(\cdot, t)\right) & \\
& +\left(\frac{\partial a_{1}}{\partial x} \theta(\cdot, t), \mathfrak{J}_{x} \oplus(\cdot, t)\right)-\left(b_{1} \theta(\cdot, t), \varpi(\cdot, t)\right)_{B_{2}^{1}(\Omega)} \\
\quad= & (f(\cdot, t, \theta(\cdot, t)), \varpi(\cdot, t))_{B_{2}^{1}(\Omega)} \quad \forall t \in I .
\end{aligned}
$$

Let $A(\theta, \omega)$ be the last three terms on the left-hand side of (4.9).

Definition 4.1. By a weak solution of problem (4.6), there exists a function $\theta: \bar{I} \rightarrow L^{2}(\Omega)$ verifying the following properties:

(i) $\theta \in L^{2}\left(I, L_{0}^{2}(\Omega)\right) \cap C\left(\bar{I}, B_{2}^{1}(\Omega)\right)$;

(ii) $\partial \theta / \partial t \in L^{2}\left(I ; B_{2}^{1}(\Omega)\right)$;

(iii) $\theta(\cdot, 0)=0$ in $B_{2}^{1}(\Omega)$;

(iv) the integral identity

$$
\left(\frac{\partial \theta(\cdot, t)}{\partial t}, \omega(\cdot, t)\right)_{B_{2}^{1}(\Omega)}+A(\theta, \varpi)=(f, \varpi)_{B_{2}^{1}(\Omega)}
$$

holds for all $\omega \in L_{0}^{2}(\Omega)$ and for all $t \in I$. 
804 Reaction-diffusion system with nonlocal conditions

We will employ the following iteration procedure:

Let $\theta_{0}=0$ and let the sequence $\left\{\theta_{n}\right\}_{n \in \mathbb{N}}$ be defined as follows: if $\theta_{n-1}$ is known, then solve

$$
\begin{gathered}
\frac{\partial \theta_{n}}{\partial t}-\frac{\partial}{\partial x}\left(a_{1}(x, t) \frac{\partial \theta_{n}}{\partial x}\right)-b_{1}(x, t) \theta_{n}=f\left(x, t, \theta_{n-1}\right) \\
\theta_{n}(x, 0)=0 \\
\int_{\Omega} x^{k} \theta_{n}(x, t) d x=0 \quad(k=0,1)
\end{gathered}
$$

for $n=1,2, \ldots$.

Section 2 implies that for fixed $n$, each of problems (4.11) possesses a unique solution. Set $z_{n}=\theta_{n+1}-\theta_{n}$. Therefore, we obtain from (4.11)

$$
\begin{gathered}
\frac{\partial z_{n}}{\partial t}-\frac{\partial}{\partial x}\left(a_{1}(x, t) \frac{\partial z_{n}}{\partial x}\right)-b_{1}(x, t) z_{n}=\Phi_{n-1}(x, t), \\
z_{n}(x, 0)=0 \\
\int_{\Omega} x^{k} z_{n}(x, t) d x=0 \quad(k=0,1),
\end{gathered}
$$

with

$$
\Phi_{n-1}(x, t)=f\left(x, t, \theta_{n}\right)-f\left(x, t, \theta_{n-1}\right) .
$$

4.3. A priori estimates. In this section, we establish estimates for the function $z_{n}$ and for its derivative with respect to time.

Considering the weak formulation of problem (4.12),

$$
\begin{aligned}
& \left(\frac{\partial z_{n}}{\partial t}(\cdot, t), \Phi(\cdot, t)\right)_{B_{2}^{1}(\Omega)}+\left(a_{1} z_{n}(\cdot, t), \oplus(\cdot, t)\right) \\
& \quad+\left(\frac{\partial a_{1}}{\partial x} z_{n}(\cdot, t), \mathfrak{J}_{x} \oplus(\cdot, t)\right)-\left(b_{1} z_{n}(\cdot, t), \oplus(\cdot, t)\right)_{B_{2}^{1}(\Omega)} \\
& \quad=\left(\Phi_{n-1}(\cdot, t), \Phi(\cdot, t)\right)_{B_{2}^{1}(\Omega)} \quad \forall t \in I .
\end{aligned}
$$

Substituting in (4.14) $\omega=z_{n}\left(\in L_{0}^{2}(\Omega)\right)$ and integrating over $(0, \tau)$, we have

$$
\begin{aligned}
& \left\|z_{n}(\cdot, \tau)\right\|_{B_{2}^{1}(\Omega)}^{2}+2 \int_{0}^{\tau}\left(a_{1} z_{n}(\cdot, t), z_{n}(\cdot, t)\right) d t \\
& =2 \int_{0}^{\tau}\left(\Phi_{n-1}(\cdot, t), z_{n}(\cdot, t)\right)_{B_{2}^{1}(\Omega)} d t-2 \int_{0}^{\tau}\left(\frac{\partial a_{1}}{\partial x} z_{n}(\cdot, t), \mathfrak{J}_{x} z_{n}(\cdot, t)\right) d t \\
& \quad+2 \int_{0}^{\tau}\left(b_{1} z_{n}(\cdot, t), z_{n}(\cdot, t)\right)_{B_{2}^{1}(\Omega)} d t .
\end{aligned}
$$


By virtue of assumption (A5') and the Cauchy inequality, equality (4.15) becomes

$$
\begin{aligned}
& c_{0} \int_{0}^{\tau}\left\|z_{n}(\cdot, t)\right\|^{2} d t+\left\|z_{n}(\cdot, \tau)\right\|_{B_{2}^{1}(\Omega)}^{2} \\
& \quad \leq \int_{0}^{\tau}\left\|\Phi_{n-1}(\cdot, t)\right\|_{B_{2}^{1}(\Omega)}^{2} d t+c_{5} \int_{0}^{\tau}\left\|z_{n}(\cdot, t)\right\|_{B_{2}^{1}(\Omega)}^{2} d t,
\end{aligned}
$$

where $c_{5}=1+c_{3}^{2} / c_{0}+2 c_{4}$.

According to a lemma of Gronwall's type, we get

$$
c_{0} \int_{0}^{\tau}\left\|z_{n}(\cdot, t)\right\|^{2} d t+\left\|z_{n}(\cdot, \tau)\right\|_{B_{2}^{1}(\Omega)}^{2} \leq \exp \left(c_{5} T\right) \int_{0}^{T}\left\|\Phi_{n-1}(\cdot, t)\right\|_{B_{2}^{1}(\Omega)}^{2} d t .
$$

The Lipschitz condition given in $\left(\mathrm{A} 5^{\prime}\right)$ leads to

$$
\int_{0}^{\tau}\left\|z_{n}(\cdot, t)\right\|^{2} d t+\left\|z_{n}(\cdot, \tau)\right\|_{B_{2}^{1}(\Omega)}^{2} \leq L^{2} \exp \left(c_{5} T\right) \int_{0}^{T}\left\|z_{n-1}(\cdot, t)\right\|_{B_{2}^{1}(\Omega)}^{2} d t
$$

Omitting the second term on the left-hand side of (4.18) and majorizing the right-hand side, it yields

$$
\left\|z_{n}\right\|_{L^{2}\left(I, L_{0}^{2}(\Omega)\right)}^{2} \leq \frac{1}{2 c_{0}} L^{2}(\beta-\alpha)^{2} \exp \left(c_{5} T\right)\left\|z_{n-1}\right\|_{L^{2}\left(I, L_{0}^{2}(\Omega)\right)}^{2}
$$

from which we get

$$
\left\|z_{n}\right\|_{L^{2}\left(I, L_{0}^{2}(\Omega)\right)} \leq L c_{6}\left\|z_{n-1}\right\|_{L^{2}\left(I, L_{0}^{2}(\Omega)\right)}
$$

where

$$
c_{6}=\frac{1}{\sqrt{2 c_{0}}} L(\beta-\alpha) \exp \left(c_{5} \frac{T}{2}\right) .
$$

On the other hand, testing identity (4.14) with $\omega=\partial z_{n} / \partial t$, we get, after some rearrangements,

$$
\begin{aligned}
2\left\|\frac{\partial z_{n}(\cdot, t)}{\partial t}\right\|_{B_{2}^{1}(\Omega)}^{2}+\frac{\partial}{\partial t}\left(a_{1} z_{n}(\cdot, t), z_{n}(\cdot, t)\right) \\
\quad=2\left(\Phi_{n-1}(\cdot, t), \frac{\partial z_{n}(\cdot, t)}{\partial t}\right)_{B_{2}^{1}(\Omega)}+\left(\frac{\partial a_{1}}{\partial t} z_{n}(\cdot, t), z_{n}(\cdot, t)\right) \\
\quad-2\left(\frac{\partial a_{1}}{\partial x} z_{n}(\cdot, t), \mathfrak{J}_{x} \frac{\partial z_{n}(\cdot, t)}{\partial t}\right)+2\left(b_{1}(\cdot, t) z_{n}(\cdot, t), \frac{\partial z_{n}(\cdot, t)}{\partial t}\right)_{B_{2}^{1}(\Omega)} .
\end{aligned}
$$


806 Reaction-diffusion system with nonlocal conditions

Integrating $(4.22)$ over $(0, \tau)$ and performing a similar computation to that of the above, it follows that

$$
\int_{0}^{\tau}\left\|\frac{\partial z_{n}(\cdot, t)}{\partial t}\right\|_{B_{2}^{1}(\Omega)}^{2} d t+\left\|z_{n}(\cdot, \tau)\right\|^{2} \leq c_{7}\left\|\Phi_{n-1}\right\|_{L^{2}\left(I ; B_{2}^{1}(\Omega)\right)}^{2}+c_{8} \int_{0}^{\tau}\left\|z_{n}(\cdot, t)\right\|^{2} d t,
$$

where $c_{7}=3 / \min \left(1, c_{0}\right), c_{8}=\left(1 / \min \left(1, c_{0}\right)\right)\left(c_{2}+3 c_{3}^{2}+\left(3(\beta-\alpha)^{2} c_{4}^{2} / 2\right)\right)$.

According to a lemma of Gronwall's type, assumption (A5'), and inequality (2.3) for $m=1$, we obtain

$$
\int_{0}^{\tau}\left\|\frac{\partial z_{n}(\cdot, t)}{\partial t}\right\|_{B_{2}^{1}(\Omega)}^{2} d t+\left\|z_{n}(\cdot, \tau)\right\|^{2} \leq c_{7} L^{2} \exp \left(c_{8} T\right)\left\|z_{n-1}\right\|_{L^{2}\left(I ; B_{2}^{1}(\Omega)\right)}^{2}
$$

Employing an elementary inequality for the norm of $z_{n-1}$ obtained from the norm of $\partial z_{n-1} / \partial t$ and omitting the second term on the left-hand side of (4.24), we get

$$
\int_{0}^{\tau}\left\|\frac{\partial z_{n}(\cdot, t)}{\partial t}\right\|_{B_{2}^{1}(\Omega)}^{2} d t \leq \frac{1}{2} c_{7} T^{2} L^{2} \exp \left(c_{8} T\right)\left\|\frac{\partial z_{n-1}}{\partial t}\right\|_{L^{2}\left(I ; B_{2}^{1}(\Omega)\right)}^{2}
$$

from which we have

$$
\left\|\frac{\partial z_{n}}{\partial t}\right\|_{L^{2}\left(I ; B_{2}^{1}(\Omega)\right)}^{2} \leq L c_{9}\left\|\frac{\partial z_{n-1}}{\partial t}\right\|_{L^{2}\left(I ; B_{2}^{1}(\Omega)\right)}^{2}
$$

where

$$
c_{9}=\sqrt{\frac{c_{7}}{2}} T \exp \left(c_{8} \frac{T}{2}\right)
$$

Thus, we have established the following result.

Theorem 4.2. Let assumptions (A4), $\left(A 5^{\prime}\right)$, and (A6) be satisfied. Then the following estimate holds:

$$
\left\|\left(z_{n}, \frac{\partial z_{n}}{\partial t}\right)\right\|_{L^{2}\left(I, L_{0}^{2}(\Omega) \times B_{2}^{1}(\Omega)\right)} \leq L c_{10}\left\|\left(z_{n-1}, \frac{\partial z_{n-1}}{\partial t}\right)\right\|_{L^{2}\left(I, L_{0}^{2}(\Omega) \times B_{2}^{1}(\Omega)\right)}
$$

for $n=1,2, \ldots$, where $c_{10}=\max \left(c_{6}, c_{9}\right)$, and $c_{6}$ and $c_{9}$ are defined by (4.21) and (4.27), respectively.

\subsection{Existence of the solution}

Theorem 4.3. There exist assumptions as in Theorem 4.2. Moreover, it is assumed that

$$
L<c_{10}^{-1}
$$


Then, there exists a function $\theta \in L^{2}\left(I, L_{0}^{2}(\Omega)\right) \cap C\left(\bar{I}, B_{2}^{1}(\Omega)\right)$ such that $\partial \theta / \partial t \in L^{2}(I$; $\left.B_{2}^{1}(\Omega)\right)$, and it satisfies

$$
\left(\theta_{n}, \frac{\partial \theta_{n}}{\partial t}\right) \underset{n \rightarrow \infty}{\longrightarrow}\left(\theta, \frac{\partial \theta}{\partial t}\right) \quad \text { in } L^{2}\left(I, L_{0}^{2}(\Omega) \times B_{2}^{1}(\Omega)\right)
$$

Proof. Observe that in inequality (4.20), if $L<1 / c_{6}$, then the series $\sum_{n} z_{n}$ and thus the sequence $\left\{S_{n}\right\}_{n}$ defined by

$$
S_{n}=\sum_{k=0}^{n-1} z_{k}=\sum_{k=0}^{n-1}\left(\theta_{k+1}(x, t)-\theta_{k}(x, t)\right)
$$

converges in $L^{2}\left(I, L_{0}^{2}(\Omega)\right)$. It is easy to see that $\theta_{n}=\theta_{0}+S_{n}=S_{n}, n=1,2, \ldots$. Therefore, the sequence $\left\{\theta_{n}\right\}_{n}$ converges in $L^{2}\left(I, L_{0}^{2}(\Omega)\right)$, that is,

$$
\theta_{n} \underset{n \rightarrow \infty}{\longrightarrow} \theta \quad \text { in } L^{2}\left(I, L_{0}^{2}(\Omega)\right)
$$

Since $L_{0}^{2}(\Omega) \hookrightarrow B_{2}^{1}(\Omega)$ (see (2.4) for $m=1$ and Remark 2.4), we have also

$$
\theta_{n} \underset{n \rightarrow \infty}{\longrightarrow} \theta \text { in } L^{2}\left(I, B_{2}^{1}(\Omega)\right)
$$

On the other hand, in inequality (4.26), if $L<1 / c_{9}$, the series $\sum_{n}\left(\partial z_{n} / \partial t\right)$ and thus the sequence $\left\{\sum_{k=0}^{n-1}\left(\left(\partial \theta_{k+1}(x, t) / \partial t\right)-\partial \theta_{k}(x, t) / \partial t\right)\right\}_{n}$ converges in $L^{2}\left(I, B_{2}^{1}(\Omega)\right)$, from which we deduce that

$$
\frac{\partial \theta_{n}}{\partial t} \underset{n \rightarrow \infty}{\longrightarrow} \psi \quad \text { in } L^{2}\left(I ; B_{2}^{1}(\Omega)\right)
$$

We have to prove that $\psi$ equals $\partial \theta / \partial t$ in $L^{2}\left(I ; B_{2}^{1}(\Omega)\right)$. To this end, we consider the identity

$$
\theta_{n}(\cdot, t)=\int_{0}^{t} \frac{\partial \theta_{n}}{\partial \tau} d \tau \quad \forall t \in I
$$

If we pass to the limit in (4.35) when $n$ tends to infinity by taking into account (4.33) and (4.34), it yields

$$
\theta(\cdot, t)=\int_{0}^{t} \psi d \tau \quad \forall t \in I, \text { in } B_{2}^{1}(\Omega),
$$

from where we conclude (see, e.g., [15, Lemma 1.3.2 and Lemma 1.3.6]) that $\theta \in C(\bar{I}$; $\left.B_{2}^{1}(\Omega)\right)$, differentiable for a.e. $t \in I$, and $\partial \theta / \partial t=\psi$ in $L^{2}\left(I ; B_{2}^{1}(\Omega)\right)$, that is

$$
\frac{\partial \theta_{n}}{\partial t} \underset{n \rightarrow \infty}{\longrightarrow} \frac{\partial \theta}{\partial t} \quad \text { in } L^{2}\left(I ; B_{2}^{1}(\Omega)\right)
$$


Consequently, for $L<c_{10}^{-1}$, the limit relation (4.30) is fulfilled. This achieves the proof of Theorem 4.3.

Theorem 4.4. Let assumptions of Theorem 4.3 be fulfilled. Then the limit function $\theta=$ $\theta(x, t)$ is the weak solution of problem (4.6) in the sense of Definition 4.1.

Proof. By virtue of Theorem 4.3, we have $\theta \in L^{2}\left(I, L_{0}^{2}(\Omega)\right) \cap C\left(\bar{I}, B_{2}^{1}(\Omega)\right)$ and $\partial \theta / \partial t \in$ $L^{2}\left(I ; B_{2}^{1}(\Omega)\right)$. Furthermore, in light of $(4.36)$, we deduce that $\theta(\cdot, 0)=0$ holds in $B_{2}^{1}(\Omega)$, and so the initial condition (4.6b) is verified. It remains to prove that $\theta$ satisfies the integral identity in Definition 4.1(iv). To this end, we consider the weak formulation of problem (4.11), and set $\theta_{n}=\left(\theta_{n}-\theta\right)+\theta$ and $f\left(x, t, \theta_{n-1}\right)=\left(f\left(x, t, \theta_{n-1}\right)-f(x, t, \theta)\right)+$ $f(x, t, \theta)$. Therefore, we get

$$
\begin{aligned}
& \left(\frac{\partial \theta_{n}(\cdot, t)}{\partial t}-\frac{\partial \theta(\cdot, t)}{\partial t}, \omega(\cdot, t)\right)_{B_{2}^{1}(\Omega)}+\left(\frac{\partial \theta(\cdot, t)}{\partial t}, \omega(\cdot, t)\right)_{B_{2}^{1}(\Omega)}+A\left(\theta_{n}-\theta, \varpi\right)+A(\theta, \varpi) \\
& =\left(f\left(\cdot, t, \theta_{n-1}(\cdot, t)\right)-f(\cdot, t, \theta(\cdot, t)), \varpi\right)_{B_{2}^{1}(\Omega)}+(f(\cdot, t, \theta), \oplus(\cdot, t))_{B_{2}^{1}(\Omega)}, \\
& \quad \forall t \in I, \forall \omega \in L_{0}^{2}(\Omega) .
\end{aligned}
$$

Thanks to the Schwarz inequality and inequality (2.3) for $m=1$, the first and third terms on the left-hand side of (4.38) and the first term on the right-hand side can be majorized as follows:

$$
\begin{aligned}
& \left(\frac{\partial \theta_{n}(\cdot, t)}{\partial t}-\frac{\partial \theta(\cdot, t)}{\partial t}, \omega(\cdot, t)\right)_{B_{2}^{1}(\Omega)} \\
& \leq\left\|\frac{\partial \theta_{n}(\cdot, t)}{\partial t}-\frac{\partial \theta(\cdot, t)}{\partial t}\right\|_{B_{2}^{1}(\Omega)}\|\omega(\cdot, t)\|_{B_{2}^{1}(\Omega)} \\
& \leq \frac{|\beta-\alpha|}{\sqrt{2}}\left\|\frac{\partial \theta_{n}(\cdot, t)}{\partial t}-\frac{\partial \theta(\cdot, t)}{\partial t}\right\|_{B_{2}^{1}(\Omega)}\|\omega(\cdot, t)\| \quad \forall t \in I, \\
& A\left(\theta_{n}-\theta, \varpi\right)=\left(a_{1}\left(\theta_{n}(\cdot, t)-\theta(\cdot, t)\right), \omega(\cdot, t)\right) \\
& +\left(\frac{\partial a_{1}}{\partial x}\left(\theta_{n}(\cdot, t)-\theta(\cdot, t)\right), \mathfrak{J}_{x} \varpi(\cdot, t)\right) \\
& -\left(b_{1}\left(\theta_{n}(\cdot, t)-\theta(\cdot, t)\right), \omega(\cdot, t)\right)_{B_{2}^{1}(\Omega)} \\
& \leq\left(c_{1}+\frac{|\beta-\alpha|}{\sqrt{2}} c_{3}+\frac{(\beta-\alpha)^{2}}{2} c_{4}\right)\left\|\theta_{n}(\cdot, t)-\theta(\cdot, t)\right\|\|\omega(\cdot, t)\| \quad \forall t \in I, \\
& \left(f\left(\cdot, t, \theta_{n-1}(\cdot, t)\right)-f(\cdot, t, \theta(\cdot, t)), \omega(\cdot, t)\right)_{B_{2}^{1}(\Omega)} \\
& \leq \frac{|\beta-\alpha|}{\sqrt{2}}\left\|f\left(\cdot, t, \theta_{n-1}(\cdot, t)\right)-f(\cdot, t, \theta(\cdot, t))\right\|_{B_{2}^{1}(\Omega)}\|\omega(\cdot, t)\| \\
& \leq \frac{(\beta-\alpha)^{2}}{2} L\left\|\theta_{n-1}(\cdot, t)-\theta(\cdot, t)\right\|\|\omega(\cdot, t)\| \quad \forall t \in I .
\end{aligned}
$$


If we pass to the limit in (4.38) when $n \rightarrow \infty$ by taking into account (4.39), (4.40), and (4.41), we obtain the integral identity

$$
\left(\frac{\partial \theta(\cdot, t)}{\partial t}, \omega(\cdot, t)\right)_{B_{2}^{1}(\Omega)}+A(\theta, \omega)=(f(\cdot, t, \theta), \omega(\cdot, t))_{B_{2}^{1}(\Omega)} \quad \forall t \in I,
$$

and the proof is complete.

4.5. Uniqueness of the solution. Assume that problem (4.6) admits two weak solutions $\theta_{1}$ and $\theta_{2}$ in $L^{2}\left(I ; L_{0}^{2}(\Omega)\right)$. Set $z=\theta_{1}-\theta_{2}$. Then $z$ satisfies

$$
\begin{gathered}
\frac{\partial z}{\partial t}-\frac{\partial}{\partial x}\left(a_{1}(x, t) \frac{\partial z}{\partial x}\right)-b_{1}(x, t) z=\Phi(x, t), \\
z(x, 0)=0 \\
\int_{\Omega} x^{k} z(x, t) d x=0 \quad(k=0,1),
\end{gathered}
$$

with

$$
\Phi(x, t)=f\left(x, t, \theta_{1}\right)-f\left(x, t, \theta_{2}\right) .
$$

Proceeding as in the establishment of estimate (4.20), we obtain

$$
\|z\|_{L^{2}\left(I ; L_{0}^{2}(\Omega)\right)} \leq L c_{6}\|z\|_{L^{2}\left(I ; L_{0}^{2}(\Omega)\right)}
$$

where $c_{6}$ is the same constant defined in (4.20). Since $L c_{6}<1$ (see (4.29)), then

$$
\|z\|_{L^{2}\left(I ; L_{0}^{2}(\Omega)\right)}=\left\|\theta_{1}-\theta_{2}\right\|_{L^{2}\left(I ; L_{0}^{2}(\Omega)\right)}=0
$$

from which we have the following theorem.

Theorem 4.5. Assume that assumptions of Theorem 4.2 are fulfilled. Then the solution of problem (4.6) is unique.

\subsection{Continuous dependence of the solution}

Theorem 4.6. If $\sigma(x, t)$ and $\sigma^{*}(x, t)$ are two solutions of problem (4.4) corresponding to $\left(\sigma_{0}, f_{4}\right)$ and $\left(\sigma_{0}^{*}, f_{4}^{*}\right)$, respectively, and if there exist a continuous nonnegative function $K(t)$ and a positive constant $L$ such that the estimate

$$
\left\|f_{4}(\cdot, t, \sigma)-f_{4}^{*}\left(\cdot, t, \sigma^{*}\right)\right\|_{B_{2}^{1}(\Omega)} \leq K(t)+L\left\|\sigma-\sigma^{*}\right\|_{B_{2}^{1}(\Omega)}
$$

holds for all $\sigma, \sigma^{*} \in B_{2}^{1}(\Omega)$ and all $t \in I$, then

$$
\left\|\sigma(\cdot, \tau)-\sigma^{*}(\cdot, \tau)\right\|_{B_{2}^{1}(\Omega)}^{2} \leq\left(\left\|\sigma_{0}-\sigma_{0}^{*}\right\|_{B_{2}^{1}(\Omega)}^{2}+\int_{0}^{\tau} K^{2}(t) d t\right) e^{c_{11} T},
$$

where $c_{11}=\exp \left(\left(1+2 L+2 c_{4}^{2}+c_{3}^{2} / 2 c_{0}\right) T\right)$. 
810 Reaction-diffusion system with nonlocal conditions

Proof. Let $s(x, t)=\sigma(x, t)-\sigma^{*}(x, t)$. Then $s(x, t)$ satisfies

$$
\begin{gathered}
\mathscr{L}_{1} s=\frac{\partial s}{\partial t}-\frac{\partial}{\partial x}\left(a_{1} \frac{\partial s}{\partial x}\right)-b_{1} s=f_{4}(x, t, \sigma)-f_{4}^{*}\left(x, t, \sigma^{*}\right) \\
\ell_{1} s=s(x, 0)=\sigma_{0}(x)-\sigma_{0}^{*}(x)=s_{0}(x) \\
\int_{\Omega} x^{k} s(x, t) d x=0, \quad k=0,1, t \in I .
\end{gathered}
$$

Considering the weak formulation of problem (4.49), letting $v=s$, and integrating by parts, we obtain, by integrating the result over $(0, \tau)$,

$$
\begin{aligned}
\|s(\cdot, \tau)\|_{B_{2}^{1}(\Omega)}^{2}+2 \int_{0}^{\tau}\left(a_{1} s(\cdot, t), s(\cdot, t)\right) d t \\
=2 \int_{0}^{\tau}\left(f_{4}(\cdot, t, \sigma)-f_{4}^{*}\left(\cdot, t, \sigma^{*}\right), s(\cdot, t)\right)_{B_{2}^{1}(\Omega)} d t+\left\|s_{0}\right\|_{B_{2}^{1}(\Omega)}^{2} \\
\quad-2 \int_{0}^{\tau}\left(\frac{\partial a_{1}}{\partial x} s(\cdot, t), \mathfrak{J}_{x} s(\cdot, t)\right) d t+2 \int_{0}^{\tau}\left(b_{1} s(\cdot, t), s(\cdot, t)\right)_{B_{2}^{1}(\Omega)} d t .
\end{aligned}
$$

Invoking assumption (A4) and (4.47) and applying the Cauchy inequality and Gronwall's lemma, we obtain inequality (4.48).

\section{Conclusion}

In this paper, we have treated a nonlinear parabolic system with only integral terms in the boundaries. We have firstly solved a similar linear problem corresponding to a single parabolic equation. Then, on the basis of the obtained results, we have constructed via an iterative process a sequence of solutions and we have proved that this sequence converges to the weak solution of the problem under study.

Our results still hold for nonlinear $2 m$-parabolic systems with only integral conditions

$$
\begin{gathered}
\mathscr{L}_{1}(u, v)=\frac{\partial u}{\partial t}+(-1)^{m} \frac{\partial^{2 m-1}}{\partial x^{2 m-1}}\left(a_{1} \frac{\partial u}{\partial x}\right)+b_{1} v=f_{1}(x, t, u, v), \\
\mathscr{L}_{2}(u, v)=\frac{\partial v}{\partial t}+(-1)^{m} \frac{\partial^{2 m-1}}{\partial x^{2 m-1}}\left(a_{2} \frac{\partial v}{\partial x}\right)+b_{2} u=f_{2}(x, t, u, v), \\
\ell_{1} u=u(x, 0)=u_{0}(x), \\
\ell_{2} v=v(x, 0)=v_{0}(x), \\
\int_{\Omega} x^{k} u(x, t) d x=m_{k}(t) \quad(k=\overline{0,2 m-1}), \\
\int_{\Omega} x^{k} v(x, t) d x=\mu_{k}(t) \quad(k=\overline{0,2 m-1}) ;
\end{gathered}
$$


by using the same method, it suffices to replace in the proofs of corresponding theorems the space $B_{2}^{1}(\Omega)$ by $B_{2}^{m}(\Omega)$.

Our results can also be extended to a mixed problem for a pluriparabolic system with integral boundary conditions (or more generally for a pluriparabolic system with nonlocal initial conditions and integral boundary conditions, resp.):

$$
\begin{aligned}
& \mathscr{L}_{1}(u, v)=\sum_{i=1}^{n} \frac{\partial u}{\partial t_{i}}+(-1)^{m} \frac{\partial^{2 m-1}}{\partial x^{2 m-1}}\left(a_{1} \frac{\partial u}{\partial x}\right)+b_{1} v=f_{1}(x, t, u, v), \\
& \mathscr{L}_{2}(u, v)=\sum_{i=1}^{n} \frac{\partial v}{\partial t_{i}}+(-1)^{m} \frac{\partial^{2 m-1}}{\partial x^{2 m-1}}\left(a_{2} \frac{\partial v}{\partial x}\right)+b_{2} u=f_{2}(x, t, u, v), \\
& \text { for } x \in \Omega, t=\left(t_{1}, \ldots, t_{n}\right) \in \prod_{i=1}^{n}\left(0, T_{i}\right) \text {, } \\
& \left(\mathscr{L}_{1}(u, v)=\sum_{i=1}^{n} \frac{\partial u}{\partial t_{i}}+(-1)^{m} \operatorname{sign} \prod_{i=1}^{n}\left(1-\left|\lambda_{i}\right|^{2}\right) \frac{\partial^{2 m-1}}{\partial x^{2 m-1}}\left(a_{1} \frac{\partial u}{\partial x}\right)+b_{1} v\right. \\
& =f_{1}(x, t, u, v) \text {, } \\
& \mathscr{L}_{2}(u, v)=\sum_{i=1}^{n} \frac{\partial v}{\partial t_{i}}+(-1)^{m} \operatorname{sign} \prod_{i=1}^{n}\left(1-\left|\lambda_{i}\right|^{2}\right) \frac{\partial^{2 m-1}}{\partial x^{2 m-1}}\left(a_{2} \frac{\partial v}{\partial x}\right)+b_{2} u \\
& \left.=f_{2}(x, t, u, v), \quad \text { for } x \in \Omega, t=\left(t_{1}, \ldots, t_{n}\right) \in \prod_{i=1}^{n}\left(0, T_{i}\right), \quad \text { resp. }\right) \text {, } \\
& \ell_{i} u=u\left(x, t_{1}, \ldots, t_{i-1}, 0, t_{i+1}, \ldots, t_{n}\right)=u_{0 i}\left(x, t_{1}, \ldots, t_{i-1}, t_{i+1}, \ldots, t_{n}\right), \\
& \ell_{i} v=v\left(x, t_{1}, \ldots, t_{i-1}, 0, t_{i+1}, \ldots, t_{n}\right)=v_{0 i}\left(x, t_{1}, \ldots, t_{i-1}, t_{i+1}, \ldots, t_{n}\right) \text {, } \\
& \left(x, t_{1}, \ldots, t_{i-1}, t_{i+1}, \ldots, t_{n}\right) \in \Omega \times \prod_{\substack{j=1 \\
j \neq i}}^{n}\left(0, T_{j}\right), \\
& \left(\ell_{i} u=u\left(x, t_{1}, \ldots, t_{i-1}, 0, t_{i+1}, \ldots, t_{n}\right)-\lambda_{i} u\left(x, t_{1}, \ldots, t_{i-1}, T_{i}, t_{i+1}, \ldots, t_{n}\right)\right. \\
& =u_{0 i}\left(x, t_{1}, \ldots, t_{i-1}, t_{i+1}, \ldots, t_{n}\right), \\
& \ell_{i} v=v\left(x, t_{1}, \ldots, t_{i-1}, 0, t_{i+1}, \ldots, t_{n}\right)-\lambda_{i} v\left(x, t_{1}, \ldots, t_{i-1}, T_{i}, t_{i+1}, \ldots, t_{n}\right) \\
& =v_{0 i}\left(x, t_{1}, \ldots, t_{i-1}, t_{i+1}, \ldots, t_{n}\right) \text {, } \\
& \left.\left(x, t_{1}, \ldots, t_{i-1}, t_{i+1}, \ldots, t_{n}\right) \in \Omega \times \prod_{\substack{j=1 \\
j \neq i}}^{n}\left(0, T_{j}\right), \quad \text { resp. }\right), \\
& \int_{\Omega} x^{k} u\left(x, t_{1}, \ldots, t_{n}\right) d x=m_{k}\left(t_{1}, \ldots, t_{n}\right), \\
& \int_{\Omega} x^{k} v\left(x, t_{1}, \ldots, t_{n}\right) d x=\mu_{k}\left(t_{1}, \ldots, t_{n}\right), \\
& \left(t_{1}, \ldots, t_{n}\right) \in \prod_{i=1}^{n}\left(0, T_{i}\right), \quad(k=\overline{0,2 m-1}) .
\end{aligned}
$$




\section{Reaction-diffusion system with nonlocal conditions}

By using the same reasoning given above, it is enough to put the test function $\sum_{i=1}^{n}\left(\partial z / \partial t_{i}\right)$ instead of $\partial z / \partial t$ and to use a generalization of Gronwall's lemma appropriate to this type problems; see, for instance, [4].

\section{References}

[1] N. Boudiba, Existence globale pour des systèmes de réaction-diffusion avec controle de masse, Ph.D. thesis, Université de Rennes I, France, 1999.

[2] A. Bouziani, Mixed problem with boundary integral conditions for a certain parabolic equation, J. Appl. Math. Stochastic Anal. 9 (1996), no. 3, 323-330.

[3] _ Mixed problems with integral conditions for certain partial differential equations, $\mathrm{Ph} . \mathrm{D}$. thesis, Constantine University, Slovakia, 1996.

[4] Strong solution for a mixed problem with nonlocal condition for certain pluriparabolic equations, Hiroshima Math. J. 27 (1997), no. 3, 373-390.

[5] - On a class of parabolic equations with a nonlocal boundary condition, Acad. Roy. Belg. Bull. Cl. Sci. (6) 10 (1999), no. 1-6, 61-77.

[6] Initial-boundary value problem with a nonlocal condition for a viscosity equation, Int. J. Math. Math. Sci. 30 (2002), no. 6, 327-338.

[7] On the quasi static flexure of a thermoelastic rod, Commun. Appl. Anal. 6 (2002), no. 4, $549-568$.

[8] On the solvability of parabolic and hyperbolic problems with a boundary integral condition, Int. J. Math. Math. Sci. 31 (2002), no. 4, 201-213.

[9] M. Dehghan, A finite difference method for a non-local boundary value problem for twodimensional heat equation, Appl. Math. Comput. 112 (2000), no. 1, 133-142.

[10] - Fully explicit finite-difference methods for two-dimensional diffusion with an integral condition, Nonlinear Anal. 48 (2002), no. 5, 637-650.

[11] A. A. Dezin, Partial Differential Equations, Springer Series in Soviet Mathematics, SpringerVerlag, Berlin, 1987.

[12] A. Haraux and A. Youkana, On a result of $K$. Masuda concerning reaction-diffusion equations, Tohoku Math. J. (2) 40 (1988), no. 1, 159-163.

[13] S. L. Hollis, R. H. Martin Jr., and M. Pierre, Global existence and boundedness in reactiondiffusion systems, SIAM J. Math. Anal. 18 (1987), no. 3, 744-761.

[14] S. L. Hollis and J. Morgan, Interior estimates for a class of reaction-diffusion systems from $L^{1}$ a priori estimates, J. Differential Equations 98 (1992), no. 2, 260-276.

[15] J. Kačur, Method of Rothe in Evolution Equations, Teubner-Texte zur Mathematik [Teubner Texts in Mathematics], vol. 80, BSB B. G. Teubner Verlagsgesellschaft, Leipzig, 1985.

[16] R. H. Martin Jr. and M. Pierre, Nonlinear reaction-diffusion systems, Nonlinear Equations in the Applied Sciences (W. F. Ames, C. Rogers, and Kapell, eds.), Math. Sci. Engrg., vol. 185, Academic Press, Massachusetts, 1992, pp. 363-398.

[17] B. Nur-Èddin and N. I. Yurchuk, A mixed problem with an integral condition for parabolic equations with a Bessel operator, Differ. Uravn. 27 (1991), no. 12, 2094-2098.

[18] C. V. Pao, Dynamics of reaction-diffusion equations with nonlocal boundary conditions, Quart. Appl. Math. 53 (1995), no. 1, 173-186.

[19] Asymptotic behavior of solutions of reaction-diffusion equations with nonlocal boundary conditions, J. Comput. Appl. Math. 88 (1998), no. 1, 225-238.

[20] Numerical solutions of reaction-diffusion equations with nonlocal boundary conditions, J. Comput. Appl. Math. 136 (2001), no. 1-2, 227-243.

[21] M. Schneider, Funktionalanalytische Methoden zur Behandlung von linearen partiellen Differentialgleichungen vom gemischten Typ, Überblicke Mathematik, Band 7, Bibliographisches Inst., Mannheim, 1974, pp. 219-265. 
[22] N. I. Yurchuk, A mixed problem with an integral condition for some parabolic equations, Differ. Uravn. 22 (1986), no. 12, 2117-2126.

Abdelfatah Bouziani: Department of Mathematics, The Larbi Ben M'hidi University Centre, P. O. Box 565, Oum El Bouaghi 04000, Algeria

E-mail address: af_bouziani@hotmail.com 


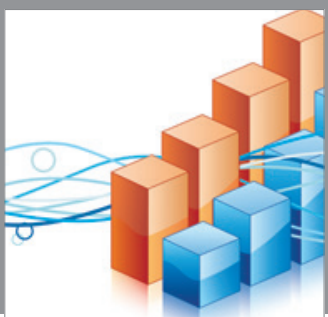

Advances in

Operations Research

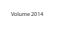

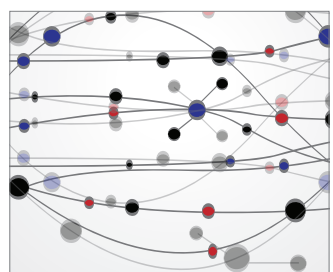

\section{The Scientific} World Journal
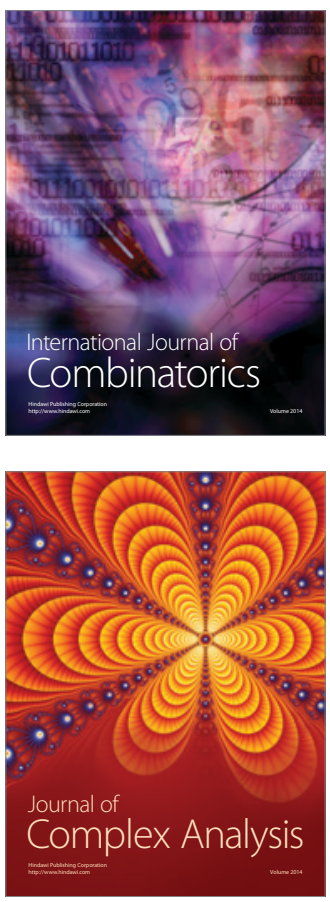

International Journal of

Mathematics and

Mathematical

Sciences
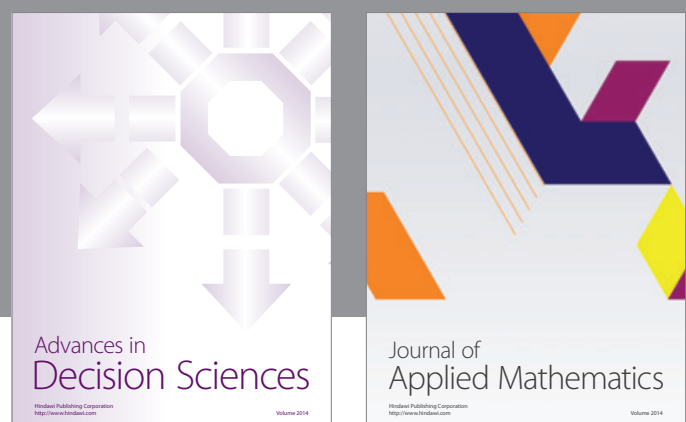

Journal of

Applied Mathematics
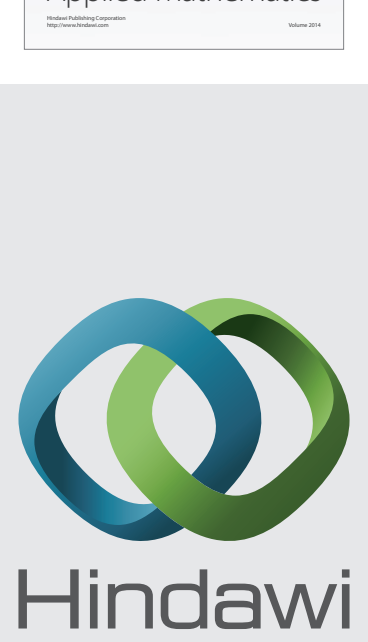

Submit your manuscripts at http://www.hindawi.com
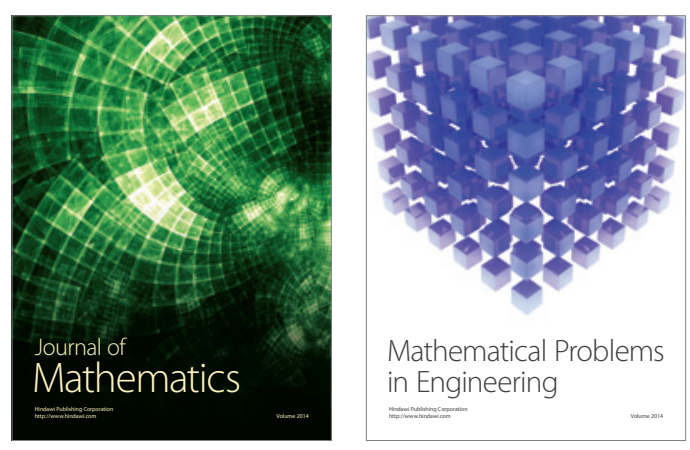

Mathematical Problems in Engineering
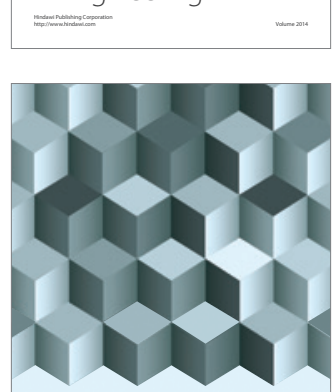

Journal of

Function Spaces
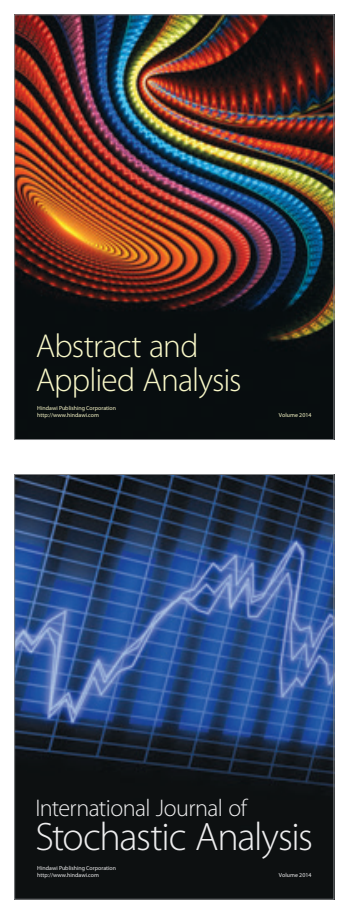

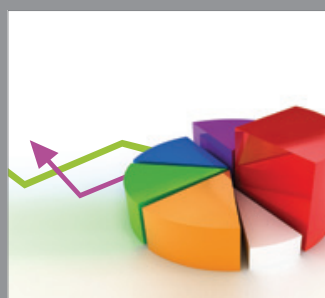

ournal of

Probability and Statistics

Promensencen
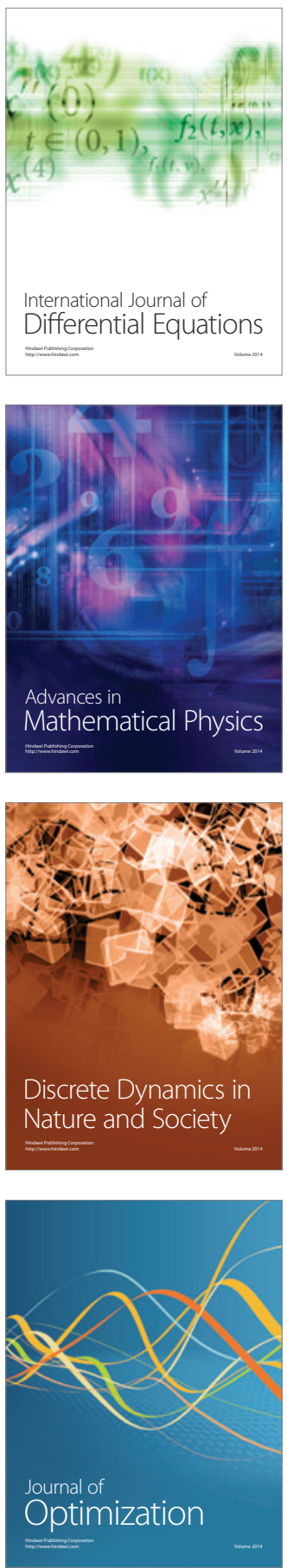